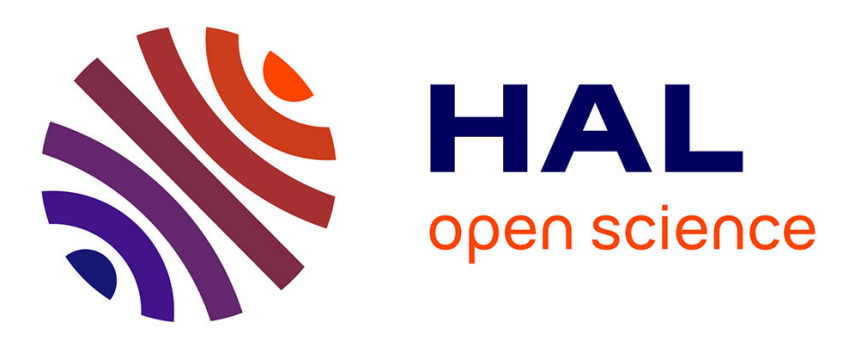

\title{
On the Modeling of the Three Types of Non-Spiking Neurons of the Caenorhabditis elegans
}

Loïs Naudin, Nathalie Corson, M. A Aziz-Alaoui, Juan Luis Jiménez Laredo, Thibaut Démare

\section{- To cite this version:}

Loïs Naudin, Nathalie Corson, M. A Aziz-Alaoui, Juan Luis Jiménez Laredo, Thibaut Démare. On the Modeling of the Three Types of Non-Spiking Neurons of the Caenorhabditis elegans. International Journal of Neural Systems, 2021, 31 (02), pp.2050063. 10.1142/S012906572050063X . hal-03351604

\author{
HAL Id: hal-03351604 \\ https://hal.science/hal-03351604
}

Submitted on 22 Sep 2021

HAL is a multi-disciplinary open access archive for the deposit and dissemination of scientific research documents, whether they are published or not. The documents may come from teaching and research institutions in France or abroad, or from public or private research centers.
L'archive ouverte pluridisciplinaire HAL, est destinée au dépôt et à la diffusion de documents scientifiques de niveau recherche, publiés ou non, émanant des établissements d'enseignement et de recherche français ou étrangers, des laboratoires publics ou privés. 


\title{
On the Modeling of the Three Types of Non-Spiking Neurons of the Caenorhabditis elegans
}

\author{
Loïs Naudin ${ }^{1}$, Nathalie Corson ${ }^{1}$, Juan Luis Jiménez Laredo ${ }^{2}$, Thibaut Démare ${ }^{2}$, and \\ M.A. Aziz-Alaoui ${ }^{1}$ \\ ${ }^{1}$ Normandie Univ, UNIHAVRE, LMAH, FR-CNRS-3335, ISCN, Le Havre 76600, France \\ ${ }^{2}$ Normandie Univ, UNIHAVRE, LITIS, FR-CNRS-3638, ISCN, Le Havre 76600, France \\ ${ }^{*}$ Corresponding author: nathalie.corson@univ-lehavre.fr
}

September 22, 2021

\begin{abstract}
The nematode Caenorhabditis elegans (C. elegans) is a well-known model organism in neuroscience. The relative simplicity of its nervous system, made up of few hundred neurons, shares some essential features with more sophisticated nervous systems, including the human one. If we are able to fully characterize the nervous system of this organism, we will be one step closer to understanding the mechanisms underlying the behavior of living things. Following a recently conducted electrophysiological survey on different $C$. elegans neurons, this paper aims at modeling the three non-spiking RIM, AIY and AFD neurons (arbitrarily named with three upper case letters by convention). To date, they represent the three possible forms of non-spiking neuronal responses of the $C$. elegans. To achieve this objective, we propose a conductance-based neuron model adapted to the electrophysiological features of each neuron. These features are based on current biological research and a series of in-silico experiments which use differential evolution to fit the model to experimental data. From the obtained results, we formulate a series of biological hypotheses regarding currents involved in the neuron dynamics. These models reproduce experimental data with a high degree of accuracy while being biologically consistent with state-of-the-art research.
\end{abstract}

Keywords: Neural system modeling; Conductance-based neuron models; Caenorhabditis elegans; Parameter estimation; Differential evolution..

\section{Introduction}

Neurosciences have not yet devised a completely satisfactory explanation of how the activity of neurons, as well as their mutual interactions, lead to the particular behavior of an organism in response to stimuli from the environment. However, understanding behavior has been a goal for researchers for the past few decades. For Sidney Brenner, to whom we owe the introduction of the nematode Caenorhabditis elegans (C. elegans) in laboratories in 1960s, behavior is "the result of a complex and ill-understood set of computations performed by nervous systems" [10]. He perceived the study of the nematode's brain as essential to understand "the way nervous systems work to produce behavior". In 1986, White et al. [84] described the full structure of the nervous system of the C. elegans, i.e. the connectome, for the first time. More than 20 years later, the team of $\mathrm{D}$. Chklovskii published an even more comprehensive version of this connectome[81].

Although the whole-brain connectomes of some other small organisms such as Drosophila melanogaster [95] or the tapdole larva of Ciona intestinalis [69] have been recently published, 
the study of $C$. elegans is still relevant due to the relative simplicity of its nervous system: the neuronal network of a hermaphrodite adult only accounts 302 neurons and about 7000 synaptic connections. Despite this, the $C$. elegans is capable of displaying a variety of complex behaviors [24] encompassing chemotaxis, the ability to regulate pharyngeal food pumping rate based on the absence or presence of food in its environment, the capacity to avoid elevated $\mathrm{CO}_{2}$ levels [11] or the ability to adapt locomotion speeding depending on food quality [75]. Furthermore, the nematode shares many of the general essential human biological features [66, 61] and, in particular, many features with the complex human nervous system using similar neurotransmitters, channels, and developmental genes [4]. In addition, some of the principles that underlie behaviors in C. elegans may also be similar in more complex animals such as humans. For instance, Chalasani et al. [15] show that both the worm olfactory system and the visual system of vertebrates follow the same general principles to process sensory information. In the same way, some information processing mechanisms are similar in other invertebrates and vertebrates brains [74]. Surprisingly, all of these features are regulated by the transmission and propagation of electrical signals along a nervous system composed of only 302 neurons. Hence, characterizing the role and activity of each of these neurons is of paramount importance to unveil the mechanisms underlying behavior.

Concerning the characterization of neurons, computational modeling is a valuable asset to investigate hypotheses not easily testable through direct experimentation thus allowing to gain biological insights into the working mechanisms of the targeted neurons [55]. Conductance-based models such as the well-known Hodgkin-Huxley model [47, 48, 49, 50, 46], as well as those derived from it [30, 45], have been widely studied in recent years [17, 7, 92, 27, 91, 38]. In simple terms, a conductance-based model is a biophysical representation of a neuron in which the ion channels are represented by conductances and the polar membrane by a capacitor. Following that line of research, this paper aims at modeling the non-spiking RIM, AIY and AFD neurons, which represent the three possible forms of non-spiking neuronal responses of the C. elegans according to Liu et al. [60]. These authors classify four large distinct classes of neurons conforming to their current-voltage (I-V) relationships as depicted in Figure 1.C. One of these classes encompasses the spiking AWA neuron which is the only one that elicits spikes. The remaining three classes comprise different types of non-spiking neurons, of which RIM, AIY and AFD neurons are representative examples. Spiking neurons compress continuous inputs into digital signals for transmitting information via action potentials. On the contrary, the non-spiking RIM, AIY and AFD neurons do not elicit action potentials and use analog signals (i.e. graded potential responses), allowing them not to sacrifice information content [73].

Highly detailed biophysical conductance-based neuron models have already been proposed for the spiking AWA neuron [60] and for the non-spiking AWC and RMD neurons [64]. However, for the three neurons under study, the lack of empirical microscopic data - detailed at the level of intervening ion currents - is an impediment to proposing the same type of modeling that is conceptualized channel by channel. Therefore, we propose a conductance-based neuron model adapted to the electrophysiological features of each neuron using the aggregated experimental membrane currents (a.k.a. total membrane currents as in Figure 1.B) and the experimental voltage traces (Figure 1.A) as references. We also take into consideration the presence or absence of plausible ion currents in each neuron from the literature and determine the intervening currents in each model from the conducted in-silico experimentation.

These series of in-silico experiments are conducted in order to estimate the parameters of each model, which is a standard approach in conductance-based modeling [87, 42, 33, 59, 41, 25, $19,80,13,14,20,62,77]$. In particular, we use differential evolution [76] which has shown to outperform genetic algorithms and simulated annealing in terms of convergence speed, simulation time, and minimization of the cost function in similar problems [12].

The remainder of the paper is organized as follows. Section 2 presents a general conductancebased neuron model to fit the membrane potential evolution of the non-spiking RIM, AIY and 
A

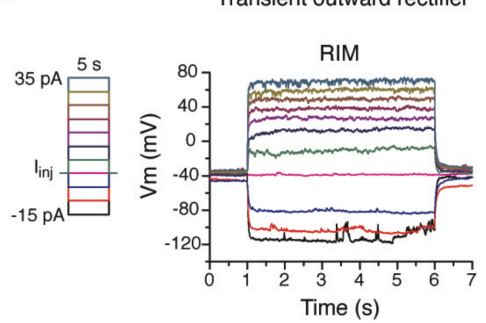

B

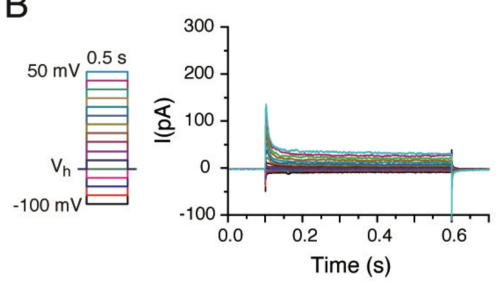

C

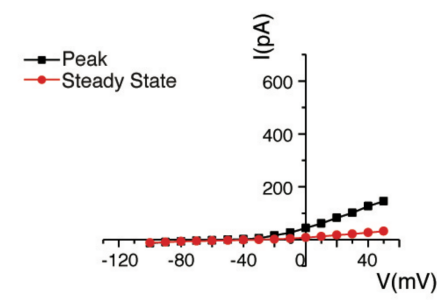

Outward rectifier

AIY
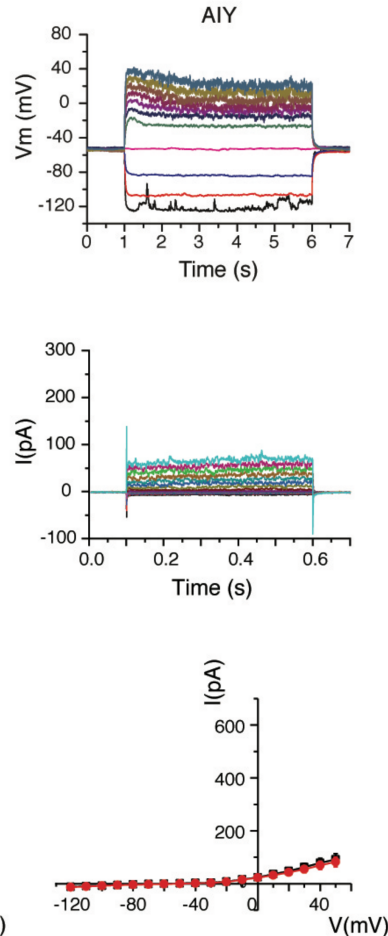

Bistable

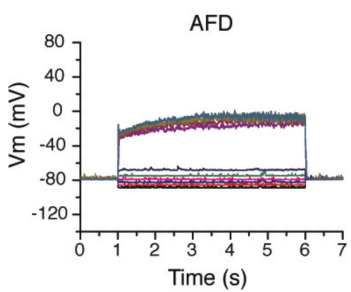

Time (s)
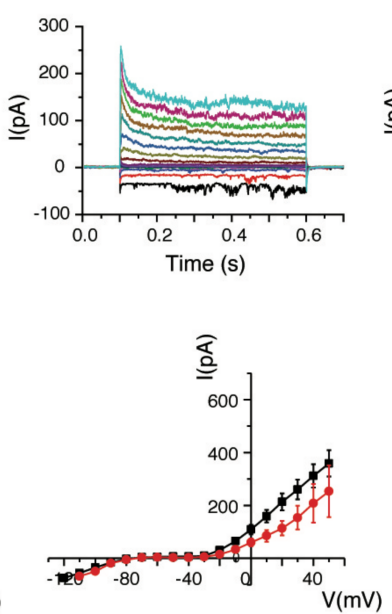

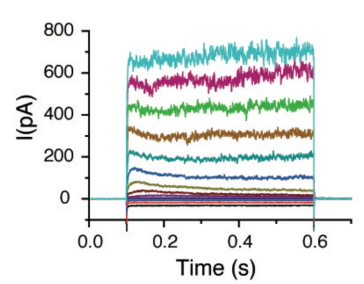

Bistable/spiking
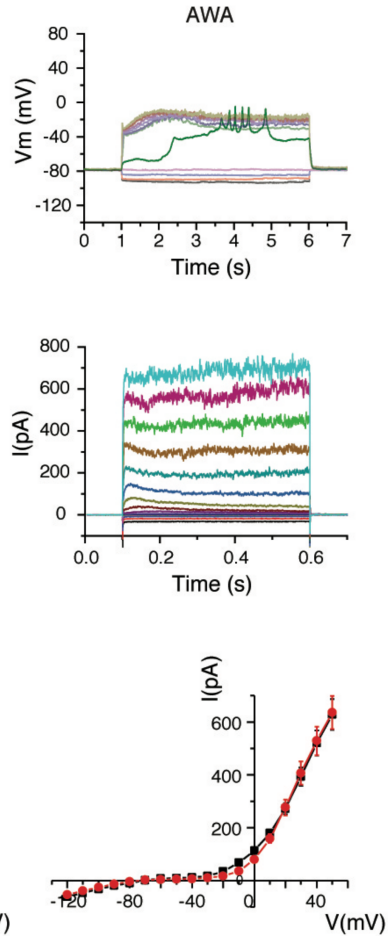

Figure 1: In-vivo recordings[60] of four different neurons of the C. elegans that represent, to date, the four forms of possible neuronal responses of the nematode. Figure A shows the evolution of the membrane potential for a series of current injections, in spans of 5 seconds, starting from $-15 \mathrm{pA}$ and increasing to $35 \mathrm{pA}$ by $5 \mathrm{pA}$ increments. Figure $\mathrm{B}$ represents the evolution of the total ion currents of the different neurons when their membrane potentials are clamped at a fixed value, in spans of 0.5 seconds, from $-100 \mathrm{mV}$ and increasing to $50 \mathrm{mV}$ by $10 \mathrm{mV}$ increments. Figure $\mathrm{C}$ describes the $\mathrm{I}-\mathrm{V}$ relationships obtained from averaged voltage-clamp recordings (RIM: $\mathrm{n}=3$; AIY: $\mathrm{n}=7$; AFD: $\mathrm{n}=3$; AWA: $\mathrm{n}=16$ ). Peak currents are measured by the absolute maximum amplitude of currents within the first $100 \mathrm{~ms}$ of each voltage step onset, while steadystate currents are measured by the averaged currents of the last $50 \mathrm{~ms}$ of each voltage step. The figure has been reproduced with the consent of the authors [60].

AFD neurons. In Section 3, we introduce the experimental setup and the methodology to conduct in-silico experiments and estimate the best parameters for the model. The obtained results are analyzed in Section 4 from a numerical and a biological perspective. Section 5 presents a discussion about the major findings of the paper. Finally, conclusions are drawn and future lines of research presented in Section 6.

\section{Model Description}

This section presents a general conductance-based neuron model for the non-spiking RIM, AIY and AFD neurons of the C. elegans. In order to fully characterize each neuron, we review the literature seeking for biological support to the plausible ion currents intervening in the neurons under study, and we present them in mathematical terms.

\section{$2.1 \quad$ General model}

Conductance-based neuron models, as the one proposed in this paper, are grounded in a series of seminal works initiated by Hodgkin and Huxley in the 1950s [47, 48, 49, 50, 46]. In general 
terms, this framework describes the neuronal dynamics in terms of activation and inactivation of voltage-gated conductances where the dynamics of the membrane potential $V$ is described by a general equation of the form

$$
C \frac{d V}{d t}=-\sum_{i o n} I_{i o n}+I
$$

where $C$ is the membrane capacitance, $d V / d t$ is the time derivative of the membrane potential, $\sum_{i o n} I_{i o n}$ is the total current flowing across the cell membrane where ion labels a generic type of ion channel, and $I$ is an input current.

The dynamics of every $I_{i o n}$ are governed by gating particles (gates) sensitive to the change in membrane potential (voltage). These gates can be of two types: activation gate and inactivation gate, each of which can be in an open or a closed state. Typically, the probability of an activation or inactivation gate being in the open state is denoted respectively by the variables $m$ and $h$ $[23,54]$. Thus, the current generated by a large population of identical ion channels is given by

$$
I_{i o n}=g_{i o n} m_{i o n}^{a} h_{i o n}^{b}\left(V-E_{i o n}\right)
$$

where $g_{i o n}$ is the maximal conductance (namely the conductance of the channel when all the gates are open); $E_{i o n}$ is the reverse potential, that is, the potential at which the ion current reverses its direction (a.k.a. equilibrium potential); and $a$ and $b$ respectively refer to the number of activation and inactivation gates. In this paper, $a$ is always assumed equal to 1 and the value of $b$ depends on features of the ionic currents. Channels that do not have inactivation gates $(b=0)$ induce a persistent current while channels that do inactivate $(b=1)$ induce a transient current.

Channels can be deactivated $(m=0)$, partially activated $(0<m<1)$, or fully activated $(m=1)$; likewise, they can be completely inactivated $(h=0)$, partially inactivated $(0<h<1)$, or deinactivated $(h=1)$. The dynamics of variables $m$ and $h$ are described by the following equation

$$
\frac{d x}{d t}=\frac{x_{\infty}(V)-x}{\tau_{x}}, x \in\{m, h\}
$$

where $\tau_{x}$ is the constant time for which $x$ reaches its respective equilibrium value $x_{\infty}$. We express $x_{\infty}$ by a Boltzmann sigmoid function [54, 23]:

$$
x_{\infty}(V)=\frac{1}{1+\exp \left(\frac{V_{1 / 2}^{x}-V}{k_{x}}\right)}, x \in\{m, h\}
$$

where $V_{1 / 2}^{x}$ satisfies $x_{\infty}\left(V_{1 / 2}^{x}\right)=1 / 2$ and $k_{x}$ is the slope factor with $k_{m}>0$ and $k_{h}<0$ as to represent activation and inactivation respectively, i.e., smaller values of $\left|k_{x}\right|$ lead to a sharper $x_{\infty}$

\subsection{Determining the $I_{i o n}$ currents involved in the model}

In order to describe the dynamics of the membrane potential of RIM, AIY and AFD neurons from equation (1), it is necessary to determine all $I_{\text {ion }}$ currents involved in the equation. Unfortunately, the three neurons under study have not yet been as thoroughly studied as, for instance, the AWA neuron for which all intervening types of currents have been studied and reported at a microscopic detail [60]. Therefore, anticipating future biological confirmation, we can only proceed by establishing a set of plausible $I_{i o n}$ currents taking into consideration some biological evidences described in the literature. Later on, in section 3, we provide a methodology for determining which of all of these plausible currents are more likely to actually intervene in each neuron. In particular, we determine the set of plausible currents based on the following evidences: 
- Goodman et al. [39] show that the outward current in C. elegans neurons is carried mostly by voltage-gated potassium channels. Furthermore, there are around 70 genes in the $C$. elegans genome that encode potassium channels $[8,72]$ which is a high number of genes compared to the size of its nervous system.

- Some papers $[16,15]$ show the existence of $C a^{2+}$ dynamics $\left(I_{C a}\right)$ in the AIY and AFD neurons, as well as in the RIM neuron [65, 94, 40]. Additionally, calcium currents have been reported in some other neurons such as AWA neuron [60], AWC neuron [15], ASER neuron [39, 58], AIB neuron [15, 40], AVA neuron [65, 94, 40] or RIA neuron [43].

- The presence of an inwardly rectifying potassium current $\left(I_{K i r}\right)$ has been experimentally confirmed in AWA neuron [60] and HSN neuron [28]. Furthermore, there are three genes that encode inward rectifier potassium channels [8] and are widely expressed in multiple neurons including sensory phasmid neurons in the tail, unidentified neurons in both the head and tail of male worms, neurons of the anterior ganglia, and different types of sensory neurons [89]. It can also be noted that the inward current for AFD (depicted in Figure 1) is typical of an inwardly rectifying potassium current.

- A leakage current corresponding to chloride channels $\left(I_{L}\right)$ is taken into account as in Refs. [60] [64] in which it plays an important role in the behavior of the neurons considered.

- C.I. Bargmann [8] shows the lack of voltage-gated $\mathrm{Na}^{+}$channels in $C$. elegans neurons.

Therefore, based on previous studies, we consider in this paper four plausible types of currents $\left(I_{K}, I_{C a}, I_{K i r}\right.$ and $\left.I_{L}\right)$ that we now describe in further detail.

\subsubsection{Potassium and calcium current}

The sequencing of the $C$. elegans genome has revealed approximately 70 genes that encode potassium channels $[8,72]$ and, consequently, a wide variety of potassium channels are expressed in one neuron, so that the total potassium current $I_{K}$ is, in reality, the sum of many potassium subcurrents. These subcurrents may differ in their activation and inactivation kinetics, activation thresholds or pharmacology. However, for the sake of simplicity and due to the lack of biological evidence about the types of potassium channels expressed in the neurons, we assume that the outward current is dominated by a unique current $I_{K}$. Likewise, the same remark can be made on the inward calcium current $I_{C a}$ : there are many different types of voltage-gated calcium channels that coexist within the cell membrane [79]. Therefore, we assume that $I_{K}$ and $I_{C a}$ respectively aggregate all the potassium and calcium subcurrents.

In addition to previous remarks, the potassium and calcium currents $\left(I_{K}\right.$ and $\left.I_{C a}\right)$ can display two different types of dynamics according to the biophysical features of neuron channels. In particular, these currents can be transient $\left(I_{i o n, t}\right)$ or persistent $\left(I_{i o n, p}\right)$ and are described by the following equations:

- Type 1. Transient current (current that inactivates):

$$
I_{\text {ion }, t}=g_{\text {ion }} m_{i o n} h_{i o n}\left(V-E_{\text {ion }}\right), \text { ion } \in\{C a, K\}
$$

- Type 2. Persistent current (current that does not inactivate):

$$
I_{\text {ion }, p}=g_{\text {ion }} m_{\text {ion }}\left(V-E_{\text {ion }}\right), \quad \text { ion } \in\{C a, K\}
$$

where the dynamic of variables $m$ and $h$ are governed by the equation (3).

The type of outward current $I_{K}$ can be determined according to the macroscopic data depicted in Figure 1. Specifically, RIM and AFD display a transient potassium current while AIY exhibits a persistent potassium current. However, we cannot identify straightforwardly the type of calcium current $I_{C a}$ due to the possible interactions occurring between different kinds of 
inward currents such as $I_{L}$ or $I_{\text {Kir }}$. Hence, as summarized in Table 1, we still need to identify whether the calcium currents are transient or persistent. In other words, in the list of plausible currents $\left(I_{K}, I_{C a}, I_{K i r}\right.$ and $\left.I_{L}\right)$, we have to split the calcium current into two possible current types: transient calcium current $\left(I_{C a, t}\right)$ and persistent calcium current $\left(I_{C a, p}\right)$.

\begin{tabular}{|l|l|l|}
\hline Neurons & RIM and AFD & AIY \\
\hline \hline Potassium current $I_{K}$ & Persistent & Transient \\
\hline Calcium current $I_{C a}$ & Unidentified & Unidentified \\
\hline
\end{tabular}

Table 1: Type of potassium $I_{K}$ and calcium $I_{C a}$ currents in RIM, AIY and AFD neurons.

\subsubsection{Leak current and inward rectifier potassium current}

The leak current $I_{L}$ from chloride $C l^{-}$channels, with constant conductance (that is, $a=b=0$ in equation (2)), is described as

$$
I_{L}=g_{L}\left(V-E_{L}\right)
$$

where $g_{L}$ is the maximal conductance of leak channels and $E_{L}$ is the reverse potential.

The inwardly rectifying potassium current $I_{K i r}$ is a current that is turned on by hyperpolarization and turned off by depolarization [44]. In the mathematical sense, this translates into the parameter $a$ being equal to $0(a=0)$ in equation (2) so that $I_{K i r}$ is inactived under depolarizations $\left(h_{\text {Kir }} \rightarrow 0\right)$ and deinactivated under hyperpolarizations $\left(h_{K i r} \rightarrow 1\right)$. Furthermore, according to [54], there are biophysical evidences showing that the kinetics of $I_{K i r}$ are practically instantaneous (that is, $\tau_{K i r}<<1$ ) so that it can be considered $h_{K i r}=h_{K i r \infty}(V)$. Therefore, this current can be modeled as

$$
I_{\text {Kir }}=g_{\text {Kir }} h_{\text {Kir } \infty}(V)\left(V-E_{K}\right) .
$$

where $h_{\text {Kir } \infty}$ is described by the equation (4)

\subsection{Summary of all possible models for each neuron}

Taking into account the list of plausible currents, we face a combinatorial problem. Until biological confirmation is obtained, rather than focusing on a single model for each neuron, we explore several possible models taking into account:

- The outward current is dominated by a potassium current: transient $\left(I_{K, t}\right)$ in the case of the RIM and AFD neurons and persistent $\left(I_{K, p}\right)$ in the case of the AIY neuron.

- There is a presence of leakage current $\left(I_{L}\right)$, mainly due to chloride channels.

- Some other types of inward currents may also be present: $I_{K i r}, I_{C a, t}$ and $I_{C a, p}$.

Considering these types of currents, Table 2 shows all the models that we are going to analyze for each neuron. In the next section we propose a numerical methodology for evaluating all of these models and for determining which of them describe the best the dynamics observed in each neuron.

\section{Methodology and Experimental Setup}

In the previous section, we determined a list of plausible models that can describe the dynamics of each neuron (see Table 2). This section proposes a methodology based on parameter estimation to determine which of the models fit best the experimental data depicted in Figure 1. Parameter estimation is carried out through differential evolution [76] using the mean squared error as estimator of the quality of the model. 


\begin{tabular}{|c|c|}
\hline RIM and AFD & AIY \\
\hline \hline$I_{K, t}+I_{L}$ & $I_{K, p}+I_{L}$ \\
\hline$I_{K i r}+I_{K, t}+I_{L}$ & $I_{K i r}+I_{K, p}+I_{L}$ \\
\hline$I_{C a, t}+I_{K, t}+I_{L}$ & $I_{C a, t}+I_{K, p}+I_{L}$ \\
\hline$I_{C a, p}+I_{K, t}+I_{L}$ & $I_{C a, p}+I_{K, p}+I_{L}$ \\
\hline$I_{C a, t}+I_{K i r}+I_{K, t}+I_{L}$ & $I_{C a, t}+I_{K i r}+I_{K, p}+I_{L}$ \\
\hline$I_{C a, p}+I_{K i r}+I_{K, t}+I_{L}$ & $I_{C a, p}+I_{K i r}+I_{K, p}+I_{L}$ \\
\hline
\end{tabular}

Table 2: List of all possible models for each neuron. Please refer to Table ?? for a full-fledged description of each one.

\subsection{Methodology}

To identify the model that best describes the dynamics of each neuron under study, a three-stage methodology is proposed, consisting of the following steps:

- Step 1. Parameter estimation:

Procedure: We have conducted 50 independent runs of the differential evolution algorithm described in section 3.2 for each of the 18 possible models listed in Table 2.

Input: 18 possible models ( 3 neurons $\times 6$ models per neuron).

Output: 50 possible parameterizations per model (900 in total).

- Step 2. Ranking the models:

Procedure: We conduct a Wittkowski statistical test (generalized Friedman rank sum test) [88] in order to produce a ranked list of the six models of every neuron.

Input: 6 models per neuron $\times 50$ parameterizations per model.

Output: The 6 models of each neuron are ranked from best to worst.

- Step 3. Selection of the best model:

Procedure: Preferentially the best ranked model is selected. However, in the case that there are no statistical differences between the best ranked model and other models, we use the peak performance measure [26] as criterion to select the best model, i.e. the parameterization with the smallest cost function prevails over the rest. This step applies pairwise comparisons using paired Wilcoxon signed-rank test [86] corrected for multiple comparisons with the Holm method [51].

Input: A list of ranked models per neuron.

Output: 1 model is selected per neuron with its corresponding parametrization.

The Wittkowski test uses the marginal likelihood principle to obtain consistent estimates for rank scores (i.e. determine which models consistently score better than others). Meanwhile, Wilcoxon signed-rank is a non-parametric test used when distributions cannot be assumed to be normally distributed. The latter test complements the former by determining whether the differences between two models with different ranks are additionally statistically different or not.

\subsection{Differential evolution (DE)}

Originally proposed by Storn and Price [76], DE is a population-based metaheuristic of the family of evolutionary algorithms capable of tackling a wide variety of problems [67, 29]. As every population-based metaheuristic, DE is an optimization method that iteratively optimizes a problem by trying to improve a set of $N P$ candidate solutions, that are initially set at random within a given solution space of $D$ parameters. New candidate solutions are generated by using 
mutation (controlled by a scaling factor $F$ ) and crossover (controlled by another factor $C R$ ) and selection then determines which of them will survive into the next generation. Although DE has undergone several improvement since its appearance [63, 21, 22], its proof of convergence [34] is based on the canonical version that we use in this paper.

When addressing parameter estimation in the context of Hodgkin-Huxley based models, DE has been shown to be an effective method and superior to other continuous optimization methods $[12,13,14]$. In these works as well as in other studies on the parametrization of $\mathrm{DE}[76,3,1,83]$, DE main parameters are fixed to $F=0.5$ and $C R=0.9$. The choice of parameters in this paper is based on these works.

We have implemented DE on Scilab by setting constraints on parameter values to remain biologically relevant (Table 3). Value ranges are determined based on literature data (e.g. see chapter 2 in [54] which lists a large number of parameter values for different models encountered in different contexts). We run the optimization process during 1000 iterations with the following parameters values: $F=0.5, C R=0.9$ and $N P=140$. The code of the algorithm is available at https://github.com/lois76/ParamEstimationDE/tree/master/differentialEvolution published under GNU Public License v3.0.

\begin{tabular}{|l|l|l|}
\hline Parameters & Min value & Max value \\
\hline \hline$g_{C a}, g_{\text {Kir }}, g_{K}, g_{L}$ & $0 n S$ & $50 n S$ \\
\hline$E_{C a}$ & $20 \mathrm{mV}$ & $150 \mathrm{mV}$ \\
\hline$E_{K}$ & $-100 \mathrm{mV}$ & $0 \mathrm{mV}$ \\
\hline$E_{L}$ & $-90 \mathrm{mV}$ & $30 \mathrm{mV}$ \\
\hline$V_{1 / 2}^{m}, V_{1 / 2}^{h}, V_{1 / 2}^{\text {Kir }}$ & $-90 \mathrm{mV}$ & $0 \mathrm{mV}$ \\
\hline$k_{m}$ & $0 \mathrm{mV}$ & $30 \mathrm{mV}$ \\
\hline$k_{h}, k_{\text {Kir }}$ & $-30 \mathrm{mV}$ & $0 \mathrm{mV}$ \\
\hline$\tau_{m}, \tau_{h}$ & $0 \mathrm{ds}$ & $15 \mathrm{ds}$ \\
\hline$x_{m}^{0}, x_{h}^{0}$ & 0 & 1 \\
\hline$C$ & 0 & 10 \\
\hline
\end{tabular}

Table 3: Parameter ranges have been obtained from literature $[54,60]$ to be biologically relevant.

\subsection{Parameter estimation}

Parameter estimation is carried out by minimizing the mean square error $(M S E)$ between the experimental voltages $V_{\text {exp }}(I, t)$ depicted in Figure 1.A and the voltages $V_{\text {est }}^{\theta}(I, t)$ estimated by the model:

$$
M S E(\theta)=\frac{1}{N} \sum_{t} \sum_{I}\left(V_{e x p}(I, t)-V_{e s t}^{\theta}(I, t)\right)^{2}
$$

where $t \in[0,50 d s]$ corresponds to the biological real time with a sampling period of $\Delta t=$ $0.004 d s ; N=12500$ is the number of data points in the measurement record; $I$ corresponds to successive step values of current injections starting from -15pA and increasing to 35pA by intervals of $5 \mathrm{pA}$; and $\theta$ is the vector containing the parameters of a specific model. For instance, the parameter vector of the $I_{K, p}+I_{L}$-model is expressed by:

$$
\theta_{I_{K, p}+I_{L}}=\left[\begin{array}{lllll}
g_{K} & g_{L} & E_{K} & E_{L} & V_{1 / 2}^{m_{K}} \\
k_{m_{K}} & \tau_{m_{K}} m_{K}^{0} C
\end{array}\right]
$$

The respective $\theta$-vectors of all possible models displayed in Table 2 are provided in Table A. We emphasize the importance of not setting the initial conditions $m^{0}$ and $h^{0}$ at any prefixed value but to consider them as parameters to be estimated, so that the algorithm can escape from bad regions in the solution space that are due to a bad choice of the initial conditions. Finally, $V_{e s t}^{\theta}(I, t)$ is calculated using the ordinary differential equation solver (ode function) of ScILAB. 


\section{Analysis of Results}

In this section, we analyze results from two complementary perspectives. First, we treat them from a global perspective. The aim is to determine which ion currents actually play a role in the functioning of the neuron. This way, we can formulate some biological hypotheses and shed some light on future biological research. Then, we provide a full-fledged characterization of the models for each neuron under study based on the selection criteria established in Section 3.1.

After conducting the 900 simulations, we rank the models using the Wittkowski test (Table 4) and perform pairwise comparisons between the different models using paired Wilcoxon signedrank test (Figure 2).

At first glance, it can be noted that none of the models yield MSE values near to zero (a MSE of zero would mean a model that perfectly fits experimental data). These results can be explained by the fact that biological measurements are not exempt from experimental noise and can also be subject to some degree of experimental error. In fact, the noise in experimental data can be easily observed in Figure 1.A where the curves present the characteristic non-smooth shape of noisy data. Additionally, we can also appreciate some fluctuations in voltage in the RIM and AIY neurons for the -15pA current injection that could well be due to measurement errors. All this implies that the experimental measurements carry an aggregated error that is reflected in the obtained results.

\begin{tabular}{|l|c|c|c|}
\hline Model & RIM & AIY & AFD \\
\hline$I_{K, t}+I_{L}$ & $5.9^{(654.7) *}$ & $/$ & $6^{(57.6) *}$ \\
\hline$I_{K, p}+I_{L}$ & $/$ & $5.8^{(181.6) *}$ & $/$ \\
\hline$I_{K i r}+I_{K, t}+I_{L}$ & $5.0^{(345.7) *}$ & $/$ & $3.7^{(17.1) *}$ \\
\hline$I_{K i r}+I_{K, p}+I_{L}$ & $/$ & $5.0^{(177.1) *}$ & $/$ \\
\hline$I_{C a, t}+I_{K, t}+I_{L}$ & $3.5^{(115.9) *}$ & $/$ & $4.1^{(17.5) *}$ \\
\hline$I_{C a, t}+I_{K, p}+I_{L}$ & $/$ & $2.4^{(65.5) *}$ & $/$ \\
\hline$I_{C a, p}+I_{K, t}+I_{L}$ & $2.2^{(69.1) *}$ & $/$ & $2.5^{(16.2)}$ \\
\hline$I_{C a, p}+I_{K, p}+I_{L}$ & $/$ & $3.3^{(72.7) *}$ & $/$ \\
\hline$I_{C a, t}+I_{K i r}+I_{K, t}+I_{L}$ & $2.8^{(64.4) *}$ & $/$ & $2.5^{(15.1)}$ \\
\hline$I_{C a, t}+I_{K i r}+I_{K, p}+I_{L}$ & $/$ & $\underline{\mathbf{1 . 2}^{(63.3)}}$ & $/$ \\
\hline$I_{C a, p}+I_{K i r}+I_{K, t}+I_{L}$ & $\underline{\mathbf{1 . 6}^{(\mathbf{5 9 . 5})}}$ & $/$ & $\mathbf{2 . 3}^{(\mathbf{1 5 . 0 )}}$ \\
\hline$I_{C a, p}+I_{K i r}+I_{K, p}+I_{L}$ & $/$ & $3.3^{(72.3) *}$ & $/$ \\
\hline
\end{tabular}

Table 4: Results of the mean rank of each model obtained using the Wittkowski test. The selected models appear in underlined bold font and have been selected according to the criteria established in Section 3.1. In parentheses the results of the best cost function (minimum mean square error (MSE)) for the different models. The $*$ stands for the models that are significantly different to the one selected (after pairwise comparisons using paired Wilcoxon signed-rank test).

From a global perspective, and according to the results displayed in Table 4 and Figure 2, we can formulate some hypotheses that should be confirmed in future biological studies. It can also be noted that some of these hypotheses - based on in-silico experiments - show consistence with current research in biology:

- Hypothesis on the RIM neuron: as a first observation, the presence of calcium currents plays a determining role in improving the results in this neuron, which is consistent with [65], [94] and [40] showing the existence of calcium dynamics in RIM. Particularly, we can presume the presence of a persistent calcium current $\left(I_{C a, p}\right)$ since the models $I_{C a, p}+$ $I_{K, t}+I_{L}(\mathrm{rank}=2.2)$ and $I_{C a, p}+I_{K i r}+I_{K, t}+I_{L}(\mathrm{rank}=1.6)$ are significantly different from $I_{C a, t}+I_{K, t}+I_{L}(\mathrm{rank}=3.5)$ and $I_{C a, t}+I_{K i r}+I_{K, t}+I_{L}(\mathrm{rank}=2.8)$ respectively. Indeed, 
the only difference between the models relies in the type of calcium current (transient vs. persistent). Additionally, the model $I_{C a, p}+I_{K i r}+I_{K, t}+I_{L}(\mathrm{rank}=1.6)$ is statistically different from $I_{C a, p}+I_{K, t}+I_{L}(\operatorname{rank}=2.2)$ which suggests the presence of $I_{K i r}$ in RIM.

- Hypothesis on the AIY neuron: the models $I_{C a, t}+I_{K, p}+I_{L}(\operatorname{rank}=2.4)$ and $I_{C a, t}+$ $I_{K i r}+I_{K, p}+I_{L}(\mathrm{rank}=1.2)$ are significantly different from $I_{C a, p}+I_{K, p}+I_{L}(\mathrm{rank}=3.3)$ and $I_{C a, p}+I_{K i r}+I_{K, p}+I_{L}(\mathrm{rank}=3.3)$ respectively, which suggest the presence of $I_{C a, t}$ in AIY. In fact, hypothesizing the presence of $I_{C a, t}$ is consistent with the experimental findings of Clark et al. [16] who observe a presence of a transient calcium current in the AIY axon. On the other hand, the model $I_{C a, t}+I_{K, p}+I_{L}(\mathrm{rank}=2.4)$ is statistically different from $I_{C a, t}+I_{K i r}+I_{K, p}+I_{L}\left(\right.$ rank=1.2) which suggests the presence of $I_{K i r}$ in AIY.

- Hypothesis on the AFD neuron: the results suggest the existence of a calcium current, which is consistent with [16]. In particular, the calcium current can be assumed of persistent type $\left(I_{C a, p}\right)$ as the models $I_{C a, p}+I_{K, t}+I_{L}(\operatorname{rank}=2.5)$ and $I_{C a, t}+I_{K, t}+I_{L}(\operatorname{rank}=4.1)$ are significantly different. We can also hypothesize the presence of an inwardly rectifying potassium current $\left(I_{K i r}\right)$ in this neuron since the models $I_{C a, t}+I_{K, t}+I_{L}(\operatorname{rank}=4.1)$ and $I_{C a, t}+I_{K i r}+I_{K, t}+I_{L}(\mathrm{rank}=2.5)$ are statistically different.

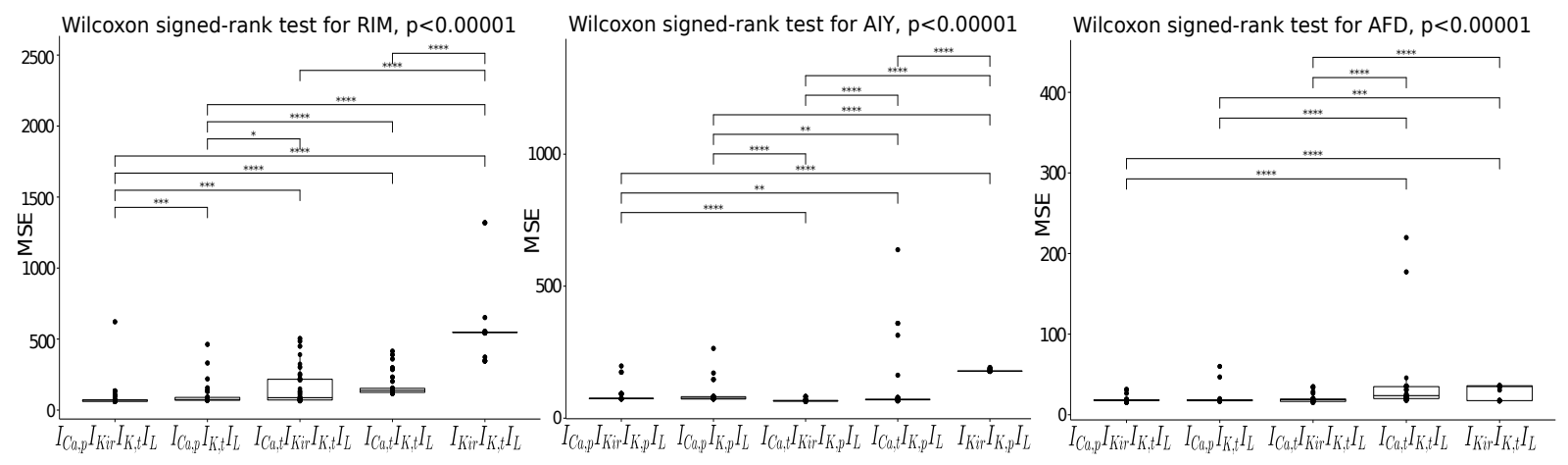

Figure 2: Pairwise comparisons using paired Wilcoxon signed-rank test and corrected for multiple comparisons with the Holm method. Connected distributions (with stars $(*)$ on top) are significantly different.

Following the previous set of hypotheses, we can proceed with the selection of the models that offer, at the same time, high accuracy and biological consistency with research for each neuron. Specifically, the models that result from the established methodology are the $I_{C a, p}+$ $I_{K i r}+I_{K, t}+I_{L}$-model for the RIM and AFD neurons, and the $I_{C a, t}+I_{K i r}+I_{K, p}+I_{L}$-model for the AIY neuron. Figure 3 shows the respective membrane potentials curves for these models (please refer to Appendix B for the associated parameter values).

In order to complete the analysis, we propose in Appendix $\mathrm{C}$ an additional metric taking into account the noise level of experimental data, from which an additional statistical analysis is conducted (Table 6). The results show consistency and reinforce the set of previously established hypotheses.

For every neuron under study, it can be observed that the curves of the models fit well with experimental data in all series of current injections. Furthermore, the quality of the fitting is maintained throughout the entire evolution of the membrane potential. In the light of these results, it can be concluded that the proposed method is robust to experimental noise and that it can be directly applied to raw experimental data. As shown in Figure 3, the proposed method converges towards models that describe the dynamics of the different neurons with good accuracy despite the experimental noise reflected in the aggregated error of the results. 

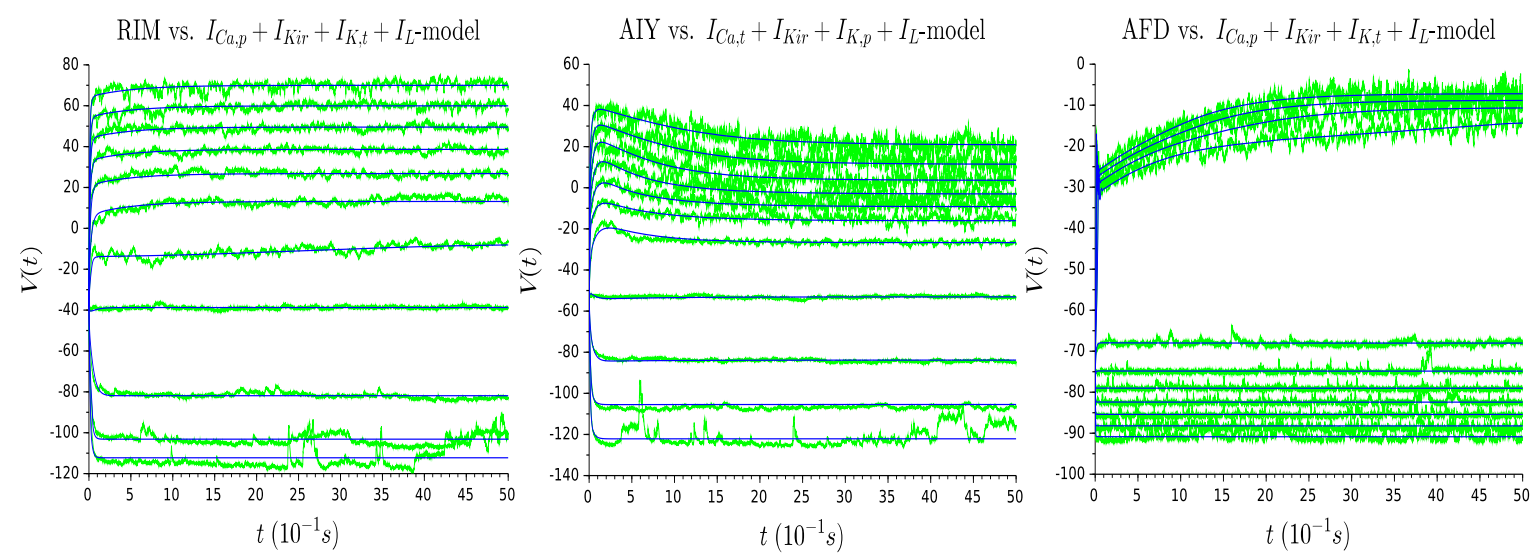

Figure 3: Evolution of membrane potential for a series of current injection starting from -15pA and increasing to $35 \mathrm{pA}$ by $5 \mathrm{pA}$ increments. Experimental data (represented in green) and outcomes of the selected models (represented in blue) overlap for the same values of current injection.

\section{Discussion}

Some experimental techniques, such as ionic conductance measures [71], are relatively hard to perform, especially in C.elegans because of the neurons' small size and the difficulty of dissecting a one milimeter long worm. These are the main reasons explaining the lack of biophysical information about $C$. elegans channels, making the current modeling of $C$. elegans neurons challenging. The advantage of the modeling methodology proposed is that it requires few experimental data for obtaining minimal deviations from empirical measurements while capturing many microscopic properties described in the literature.

In the light of the obtained results, we have formulated a series of biological hypotheses. In particular, the results suggest, on the one hand, the presence of a calcium current in all the neurons, which is consistent with reported cases in the literature and, on the other hand, its important role in the quality of the fitting. More in detail, the results point out a persistent type calcium current $\left(I_{C a, p}\right)$ for the RIM and AFD neurons and a transient type calcium current $\left(I_{C a, t}\right)$ for AIY, which is consistent with other works in the literature tackling the latter neuron. Results also indicate the presence of an inwardly rectifying potassium current $\left(I_{K i r}\right)$ in the three neurons. That way, the $I_{C a, p}+I_{K i r}+I_{K, t}+I_{L}$-model for the RIM and AFD neurons, and the $I_{C a, t}+I_{K i r}+I_{K, p}+I_{L}$-model for the AIY neuron are able to reproduce experimental data with a high degree of accuracy while being biologically consistent with state-of-the-art research.

Although our formulated hypotheses should be validated in future electrophysiological experiments, we argue that the models and mathematical framework presented in this paper provide a valuable asset for the modeling and study of the nematode neural network under more realistic electrophysiological models. Indeed, the non-spiking neural network of the C. elegans is a relevant subject of study [85, 70, 68, 56, 57, 18].

In order to expand the method proposed in this paper to other contexts such as spiking neurons, an essential step would be to define feature-based errors functions[25] more adapted than the MSE for quantitatively capturing features relative to spiking pattern, as in [82] and [53]. This line of research can be promising as spiking neural networks are the most widely studied case in the literature $[9,31,52,78,93,32,6,90,5,36,37,35,2]$. 


\section{Conclusions}

In this paper, we have presented a conductance-based model for the RIM, AIY and AFD nonspiking neurons of the $C$. elegans along with a methodology for determining the plausible intervening currents in these neurons due to the lack of electrophysiological data. Results suggest the presence of an inwardly rectifying potassium current $\left(I_{\text {Kir }}\right)$ in every neuron as well as the existence of $\mathrm{Ca}^{2+}$ dynamics $\left(I_{C a}\right)$ that depending on the neuron can be of transient type (e.g. $\mathrm{AIY}$ ) or persistent type (e.g. RIM and AFD). Although the existence of these currents is yet to be demonstrated, the respective formulated models are able to reproduce the evolution of the membrane potential with good accuracy. In the future, the following natural step is to pursue the validation of these hypotheses from electrophysiological studies.

\section{Acknowledgements}

This work was supported by the ERDF (XTerm Project), Normandie Region (France) and by The LMAH, FR-CNRS-3335. We thank Qiang Liu, Frank Le Foll, Aurélien Chatelier and Hélène Castel for their advice on the biological aspects of this paper, and Carlos M. Fernandes, Nuno Fachada and Delphine Grancher for the discussions on the statistical part. Thanks to the reviewers, their comments were of valuable help in improving the quality of the paper.

\section{A Full-fledged material description}

\begin{tabular}{|c|c|}
\hline \multirow[t]{2}{*}{$\begin{array}{l}I_{K, p}+I_{L^{-}} \\
\text {model }\end{array}$} & $\left\{\begin{array}{l}C \dot{V}=-g_{K} m_{K}\left(V-E_{K}\right)-g_{L}\left(V-E_{L}\right)+I \\
\tau_{m_{K}} m_{K}=m_{K \infty}(V)-m_{K}\end{array}\right.$ \\
\hline & $\theta=\left[\begin{array}{lllllll}g_{K} & g_{L} & E_{K} & E_{L} & V_{1 / 2}^{m_{K}} & k_{m_{K}} & \tau_{m_{K}} \\
m_{K}^{0} & C\end{array}\right]$ \\
\hline \multirow[t]{2}{*}{$\begin{array}{l}I_{K, t}+I_{L^{-}} \\
\text {model }\end{array}$} & $\left\{\begin{array}{l}C \dot{V}=-g_{K} m_{K} h_{K}\left(V-E_{K}\right)-g_{L}\left(V-E_{L}\right)+I \\
\tau_{m_{K}} m_{K}=m_{K \infty}(V)-m_{K} \\
\tau_{h_{K}} h_{K}=h_{K \infty}(V)-h_{K}\end{array}\right.$ \\
\hline & 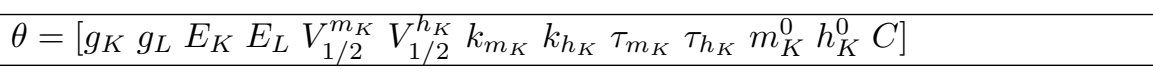 \\
\hline \multirow[t]{2}{*}{$\begin{array}{l}I_{K i r}+I_{K, p}+ \\
I_{L} \text {-model }\end{array}$} & $\left\{\begin{array}{l}C \dot{V}=g_{K i r} h_{K i r \infty}(V)\left(V-E_{K}\right)-g_{K} m_{K}\left(V-E_{K}\right)-g_{L}\left(V-E_{L}\right)+I \\
\tau_{m_{K}} m_{K}=m_{K \infty}(V)-m_{K}\end{array}\right.$ \\
\hline & 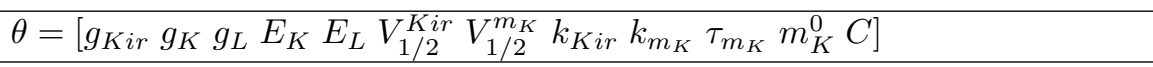 \\
\hline \multirow[t]{2}{*}{$\begin{array}{l}I_{K i r}+I_{K, t}+ \\
I_{L} \text {-model }\end{array}$} & $\left\{\begin{array}{l}C \dot{V}=g_{K i r} h_{K i r \infty}(V)\left(V-E_{K}\right)-g_{K} m_{K} h_{K}\left(V-E_{K}\right)-g_{L}\left(V-E_{L}\right)+I \\
\tau_{m_{K}} \dot{m}_{K}=m_{K \infty}(V)-m_{K} \\
\tau_{h_{K}} h_{K}=h_{K \infty}(V)-h_{K}\end{array}\right.$ \\
\hline & 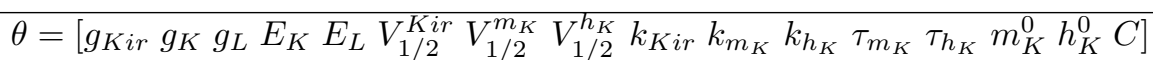 \\
\hline
\end{tabular}




\begin{tabular}{|c|c|}
\hline \multirow[t]{2}{*}{$\begin{array}{l}I_{C a, t}+ \\
I_{K, t}+I_{L^{-}} \\
\text {model }\end{array}$} & $\left\{\begin{array}{l}C \dot{V}=-g_{C a} m_{C a} h_{C a}\left(V-E_{C a}\right)-g_{K} m_{K} h_{K}\left(V-E_{K}\right)-g_{L}\left(V-E_{L}\right)+I \\
\tau_{m_{C a}} m_{C a}=m_{C a \infty}(V)-m_{C a} \\
\tau_{h_{C a}} h_{C a}=h_{C a \infty}(V)-h_{C a} \\
\tau_{m_{K}} \dot{m}_{K}=m_{K \infty}(V)-m_{K} \\
\tau_{h_{K}} \cdot \dot{h_{K}}=h_{K \infty}(V)-h_{K}\end{array}\right.$ \\
\hline & 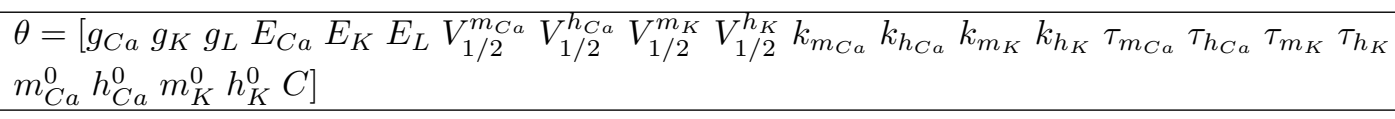 \\
\hline \multirow[t]{2}{*}{$\begin{array}{l}I_{C a, t}+ \\
I_{K, p}+I_{L^{-}} \\
\text {model }\end{array}$} & $\left\{\begin{array}{l}C \dot{V}=-g_{C a} m_{C a} h_{C a}\left(V-E_{C a}\right)-g_{K} m_{K}\left(V-E_{K}\right)-g_{L}\left(V-E_{L}\right)+I \\
\tau_{m_{C a}} m_{C a}=m_{C a \infty}(V)-m_{C a} \\
\tau_{h_{C a}} h_{C a}=h_{C a \infty}(V)-h_{C a} \\
\tau_{m_{K}} m_{K}=m_{K \infty}(V)-m_{K}\end{array}\right.$ \\
\hline & 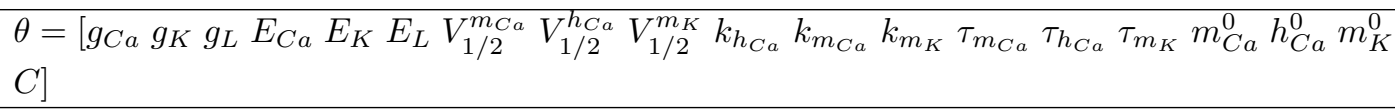 \\
\hline \multirow[t]{2}{*}{$\begin{array}{l}I_{C a, p}+ \\
I_{K, t}+I_{L^{-}} \\
\text {model }\end{array}$} & $\left\{\begin{array}{l}C \dot{V}=-g_{C a} m_{C a}\left(V-E_{C a}\right)-g_{K} m_{K} h_{K}\left(V-E_{K}\right)-g_{L}\left(V-E_{L}\right)+I \\
\tau_{m_{C a}} m_{C a}=m_{C a \infty}(V)-m_{C a} \\
\tau_{m_{K}} \dot{m}_{K}=m_{K \infty}(V)-m_{K} \\
\tau_{h_{K}} h_{K}=h_{K \infty}(V)-h_{K}\end{array}\right.$ \\
\hline & 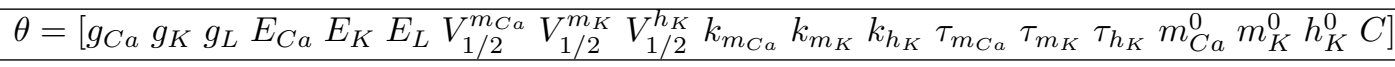 \\
\hline \multirow[t]{2}{*}{$\begin{array}{l}I_{C a, p}+ \\
I_{K, p}+I_{L^{-}} \\
\text {model }\end{array}$} & $\left\{\begin{array}{l}C \dot{V}=-g_{C a} m_{C a}\left(V-E_{C a}\right)-g_{K} m_{K}\left(V-E_{K}\right)-g_{L}\left(V-E_{L}\right)+I \\
\tau_{m_{C a}} m_{C a}=m_{C a \infty}(V)-m_{C a} \\
\tau_{m_{K}} m_{K}=m_{K \infty}(V)-m_{K}\end{array}\right.$ \\
\hline & 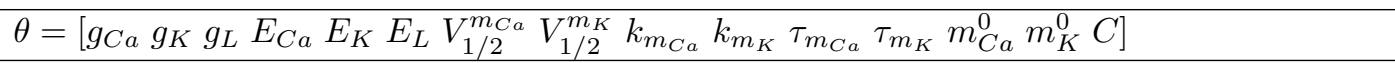 \\
\hline
\end{tabular}




\begin{tabular}{|c|c|}
\hline \multirow[t]{2}{*}{$\begin{array}{ll}I_{C a, t} & + \\
I_{K i r} & + \\
I_{K, t} & + \\
\text { model } & I_{L^{-}}\end{array}$} & $\left\{\begin{array}{l}C \dot{V}=-g_{C a} m_{C a} h_{C a}\left(V-E_{C a}\right)-g_{K i r} h_{K i r \infty}(V)\left(V-E_{K}\right)-g_{K} m_{K} h_{K}\left(V-E_{K}\right)-g_{L}\left(V-E_{L}\right)+I \\
\tau_{m_{C a}} m_{C a}=m_{C a \infty}(V)-m_{C a} \\
\tau_{h_{C a}} h_{C a}=h_{C a \infty}(V)-h_{C a} \\
\tau_{m_{K}} m_{K}=m_{K \infty}(V)-m_{K} \\
\tau_{h_{K}} \cdot \dot{h_{K}}=h_{K \infty}(V)-h_{K}\end{array}\right.$ \\
\hline & 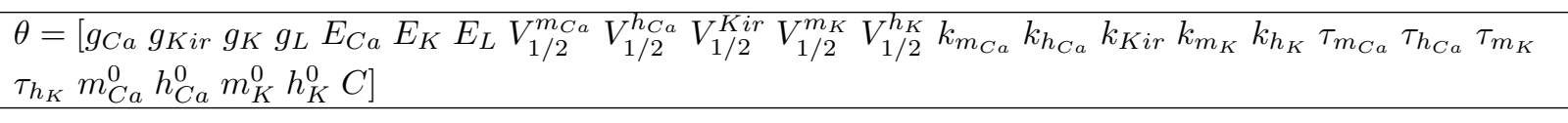 \\
\hline \multirow[t]{2}{*}{$\begin{array}{ll}I_{C a, t} & + \\
I_{K i r} & + \\
I_{K, p}+ & I_{L^{-}} \\
\text {model } & \end{array}$} & $\left\{\begin{array}{l}C \dot{V}=-g_{C a} m_{C a} h_{C a}\left(V-E_{C a}\right)-g_{K i r} h_{K i r \infty}(V)\left(V-E_{K}\right)-g_{K} m_{K}\left(V-E_{K}\right)-g_{L}\left(V-E_{L}\right)+I \\
\tau_{m_{C a}} m_{C a}=m_{C a \infty}(V)-m_{C a} \\
\tau_{h_{C a}} h_{C a}=h_{C a \infty}(V)-h_{C a} \\
\tau_{m_{K}} m_{K}=m_{K \infty}(V)-m_{K}\end{array}\right.$ \\
\hline & $\begin{array}{l}\theta=\left[g_{C a} g_{K i r} g_{K} g_{L} E_{C a} E_{K} E_{L} V_{1 / 2}^{m_{C a}} V_{1 / 2}^{h_{C a}} V_{1 / 2}^{K i r} V_{1 / 2}^{m_{K}} k_{m_{C a}} k_{h_{C a}} k_{K i r} k_{m_{K}} \tau_{m_{C a}} \tau_{h_{C a}} \tau_{m_{K}} m_{C a}^{0} h_{C a}^{0}\right. \\
\left.m_{K}^{0} C\right]\end{array}$ \\
\hline \multirow[t]{2}{*}{$\begin{array}{lr}I_{C a, p} & + \\
I_{K i r} & + \\
I_{K, t} & + \\
\text { model } & I_{L^{-}}\end{array}$} & $\left\{\begin{array}{l}C \dot{V}=-g_{C a} m_{C a}\left(V-E_{C a}\right)-g_{K i r} h_{K i r \infty}(V)\left(V-E_{K}\right)-g_{K} m_{K} h_{K}\left(V-E_{K}\right)-g_{L}\left(V-E_{L}\right)+I \\
\tau_{m_{C a}} m_{C a}=m_{C a \infty}(V)-m_{C a} \\
\tau_{m_{K}} \dot{m_{K}}=m_{K \infty}(V)-m_{K} \\
\tau_{h_{K}} h_{K}=h_{K \infty}(V)-h_{K}\end{array}\right.$ \\
\hline & $\begin{array}{l}\theta=\left[g_{C a} g_{K i r} g_{K} g_{L} E_{C a} E_{K} E_{L} V_{1 / 2}^{m_{C a}} V_{1 / 2}^{K i r} V_{1 / 2}^{m_{K}} V_{1 / 2}^{h_{K}} k_{m_{C a}} k_{K i r} k_{m_{K}} k_{h_{K}} \tau_{m_{C a}} \tau_{m_{K}} \tau_{h_{K}} m_{C a}^{0} m_{K}^{0} h_{K}^{0}\right. \\
C]\end{array}$ \\
\hline \multirow[t]{2}{*}{$\begin{array}{lr}I_{C a, p} & + \\
I_{K i r} & + \\
I_{K, p} & + \\
\text { model } & I_{L^{-}}\end{array}$} & $\left\{\begin{array}{l}C \dot{V}=-g_{C a} m_{C a}\left(V-E_{C a}\right)-g_{K i r} h_{K i r \infty}(V)\left(V-E_{K}\right)-g_{K} m_{K}\left(V-E_{K}\right)-g_{L}\left(V-E_{L}\right)+I \\
\tau_{m_{C a}} m_{C a}=m_{C a \infty}(V)-m_{C a} \\
\tau_{m_{K}} m_{K}=m_{K \infty}(V)-m_{K}\end{array}\right.$ \\
\hline & $\theta=\left[\begin{array}{ll}g_{C a} g_{K i r} & \left.g_{K} g_{L} E_{C a} E_{K} E_{L} V_{1 / 2}^{m_{C a}} V_{1 / 2}^{K i r} V_{1 / 2}^{m_{K}} k_{m_{C a}} k_{K i r} k_{m_{K}} \tau_{m_{C a}} \tau_{m_{K}} m_{C a}^{0} m_{K}^{0} C\right]\end{array}\right]$ \\
\hline
\end{tabular}




\section{B Estimated parameters for the selected models in each of the neurons}

Table 5 shows the estimated parameters for the selected models in each of the neurons.

\begin{tabular}{|l|l|l|l|}
\hline \multirow{2}{*}{ Paramètres } & \multicolumn{3}{|c|}{ Neurones } \\
\cline { 2 - 4 } & RIM & \multicolumn{1}{|c|}{ AIY } & AFD \\
\hline$g_{C a}$ & 0.24 & 0.746 & 0.1 \\
\hline$g_{K i r}$ & 0.332 & 0.1 & 1.92 \\
\hline$g_{K}$ & 0.127 & 0.17 & 12.62 \\
\hline$g_{L}$ & 0.28 & 0.2 & 0.1 \\
\hline$E_{C a}$ & 105.3 & 63.33 & 144.38 \\
\hline$E_{K}$ & -100 & -99.9 & -83.7 \\
\hline$E_{L}$ & -81.3 & -58.76 & -63.27 \\
\hline$V_{1 / 2}^{m_{C a}}$ & -21.04 & -2.31 & -16.34 \\
\hline$V_{1 / 2}^{h_{C a}}$ & & -44.13 & \\
\hline$V_{1 / 2}^{h_{K i r}}$ & -89.99 & -89.8 & -67.44 \\
\hline$V_{1 / 2}^{m_{K}}$ & -17.7 & -10.5 & -3.31 \\
\hline$V_{1 / 2}^{h_{K}}$ & -21.28 & & -65.4 \\
\hline$k_{m_{C a}}$ & 28.8 & 13.48 & 1.84 \\
\hline$k_{h_{C a}}$ & & -21.47 & \\
\hline$k_{h_{K i r}}$ & -1.2 & -3.77 & -11.46 \\
\hline$k_{m_{K}}$ & 1.18 & 7.95 & 7.26 \\
\hline$k_{h_{K}}$ & -4.64 & & -29.5 \\
\hline$\tau_{m_{C a}}$ & 0.16 & 0.33 & 6.64 \\
\hline$\tau_{h_{C a}}$ & & 9.31 & \\
\hline$\tau_{m_{K}}$ & 0.2 & 0.002 & 0.082 \\
\hline$\tau_{h_{K}}$ & 5.08 & - & 3.63 \\
\hline$m_{C a}^{0}$ & 0.349 & 0.04 & 0.002 \\
\hline$h_{C a}^{0}$ & & 0.52 & \\
\hline$m_{K}^{0}$ & 0.79 & 0.34 & 0.001 \\
\hline$h_{K}^{0}$ & 0.13 & & 0.991 \\
\hline$C$ & 0.02 & 0.028 & 0.049 \\
\hline & & & \\
\hline
\end{tabular}

Table 5: Estimated parameters for the selected models in each of the neurons, i.e. $I_{C a, p}+I_{K i r}+$ $I_{K, t}+I_{L}$-model for RIM and AFD, and $I_{C a, t}+I_{K i r}+I_{K, p}+I_{L}$-model for AIY. The estimated parameters for the rest of models are available at this github repository. 


\section{Alternative metric}

An additional metric $F$ is used to complete the analysis by normalizing the results to the deviation of each experimental voltage trace:

$$
F(\theta)=\frac{1}{|I|} \sum_{I} \frac{\sqrt{\frac{1}{N} \sum_{t}\left(V_{e x p}(I, t)-V_{e s t}^{\theta}(I, t)\right)^{2}}}{\sigma_{I}}
$$

where $\sigma_{I}$, estimated as in [87], is the noise level (i.e. standard deviation) for the entire voltage trace associated with the injection current $I$.

\begin{tabular}{|l|c|c|c|}
\hline Model & RIM & AIY & AFD \\
\hline$I_{K, t}+I_{L}$ & $6^{(3.8) *}$ & $/$ & $6^{(1.85) *}$ \\
\hline$I_{K, p}+I_{L}$ & $/$ & $6^{(2.06) *}$ & $/$ \\
\hline$I_{K i r}+I_{K, t}+I_{L}$ & $4.9^{(2.4) *}$ & $/$ & $3.8^{(0.98) *}$ \\
\hline$I_{K i r}+I_{K, p}+I_{L}$ & $/$ & $4.8^{(1.7) *}$ & $/$ \\
\hline$I_{C a, t}+I_{K, t}+I_{L}$ & $4.0^{(1.43) *}$ & $/$ & $4.6^{(1.04) *}$ \\
\hline$I_{C a, t}+I_{K, p}+I_{L}$ & $/$ & $2.3^{(1.17) *}$ & $/$ \\
\hline$I_{C a, p}+I_{K, t}+I_{L}$ & $2.5^{(1.31) *}$ & $/$ & $2.6^{(0.97) *}$ \\
\hline$I_{C a, p}+I_{K, p}+I_{L}$ & $/$ & $3.9^{(1.4) *}$ & $/$ \\
\hline$I_{C a, t}+I_{K i r}+I_{K, t}+I_{L}$ & $2.6^{(1.19) *}$ & $/$ & $2.8^{(0.958) *}$ \\
\hline$I_{C a, t}+I_{K i r}+I_{K, p}+I_{L}$ & $/$ & $\underline{\mathbf{1 . 0}^{(\mathbf{1 . 1 1})}}$ & $/$ \\
\hline$I_{C a, p}+I_{K i r}+I_{K, t}+I_{L}$ & $\underline{\mathbf{1 . 1}^{(\mathbf{1 . 1 1})}}$ & $/$ & $\underline{\mathbf{1 . 3}^{(\mathbf{0 . 9 5 2})}}$ \\
\hline$I_{C a, p}+I_{K i r}+I_{K, p}+I_{L}$ & $/$ & $3.0^{(1.24) *}$ & $/$ \\
\hline
\end{tabular}

Table 6: Results of the mean rank of each model obtained using the Wittkowski test. The selected models appear in underlined bold font and have been selected according to the criteria established in Section 3.1. In parentheses the results of the best cost function $F$ (10) for the different models. The $*$ stands for the models that are significantly different to the one selected (after pairwise comparisons using paired Wilcoxon signed-rank test).

\section{References}

[1] AA Abou El Ela, MA Abido, and SR Spea. Optimal power flow using differential evolution algorithm. Electric Power Systems Research, 80(7):878-885, 2010.

[2] Hojjat Adeli and Samanwoy Ghosh-Dastidar. Automated EEG-based diagnosis of neurological disorders: Inventing the future of neurology. CRC press, 2010.

[3] M Montaz Ali and A Törn. Population set-based global optimization algorithms: some modifications and numerical studies. Computers \& Operations Research, 31(10):1703-1725, 2004 .

[4] Z.F. Altun, L.A. Herndon, C.A. Wolkow, C. Crocker, R Lints, and D.H. Hall. Wormatlas. http://www.wormatlas.org, 2002-2020.

[5] Alberto Antonietti, Jessica Monaco, Egidio D'Angelo, Alessandra Pedrocchi, and Claudia Casellato. Dynamic redistribution of plasticity in a cerebellar spiking neural network reproducing an associative learning task perturbed by tms. International journal of neural systems, 28(09):1850020, 2018.

[6] Gabriela Antunes, Samuel F Faria da Silva, and Fabio M Simoes de Souza. Mirror neurons modeled through spike-timing-dependent plasticity are affected by channelopathies 
associated with autism spectrum disorder. International journal of neural systems, 28(05): $1750058,2018$.

[7] Aymen Balti, Valentina Lanza, and Moulay Aziz-Alaoui. A multi-base harmonic balance method applied to hodgkin-huxley model. Mathematical Biosciences $\&$ Engineering, 15(3): $807,2018$.

[8] Cornelia I Bargmann. Neurobiology of the caenorhabditis elegans genome. Science, 282 (5396):2028-2033, 1998.

[9] Marie Bernert and Blaise Yvert. An attention-based spiking neural network for unsupervised spike-sorting. International journal of neural systems, 29(08):1850059, 2019.

[10] Sydney Brenner. The genetics of caenorhabditis elegans. Genetics, 77(1):71-94, 1974.

[11] Andrew Jonathan Bretscher, Karl Emanuel Busch, and Mario de Bono. A carbon dioxide avoidance behavior is integrated with responses to ambient oxygen and food in caenorhabditis elegans. Proceedings of the National Academy of Sciences, 105(23):8044-8049, 2008.

[12] Laure Buhry, Sylvain Saighi, Audrey Giremus, Eric Grivel, and Sylvie Renaud. Parameter estimation of the hodgkin-huxley model using metaheuristics: application to neuromimetic analog integrated circuits. In 2008 IEEE Biomedical Circuits and Systems Conference, pages 173-176. IEEE, 2008.

[13] Laure Buhry, Filippo Grassia, Audrey Giremus, Eric Grivel, Sylvie Renaud, and Sylvain Saïghi. Automated parameter estimation of the hodgkin-huxley model using the differential evolution algorithm: application to neuromimetic analog integrated circuits. Neural computation, 23(10):2599-2625, 2011.

[14] Laure Buhry, Michele Pace, and Sylvain Saïghi. Global parameter estimation of an hodgkinhuxley formalism using membrane voltage recordings: application to neuro-mimetic analog integrated circuits. Neurocomputing, 81:75-85, 2012.

[15] Sreekanth H Chalasani, Nikos Chronis, Makoto Tsunozaki, Jesse M Gray, Daniel Ramot, Miriam B Goodman, and Cornelia I Bargmann. Dissecting a circuit for olfactory behaviour in caenorhabditis elegans. Nature, 450(7166):63, 2007.

[16] Damon A Clark, David Biron, Piali Sengupta, and Aravinthan DT Samuel. The afd sensory neurons encode multiple functions underlying thermotactic behavior in caenorhabditis elegans. Journal of Neuroscience, 26(28):7444-7451, 2006.

[17] Nathalie Corson, Valentina Lanza, and Nathalie Verdière. Hopf bifurcations in directed acyclic networks of linearly coupled hindmarsh-rose systems. Acta biotheoretica, 64(4): 375-402, 2016.

[18] Alicia Costalago-Meruelo, Pedro Machado, Kofi Appiah, Andoni Mujika, Peter Leskovsky, Roberto Alvarez, Gorka Epelde, and T Martin McGinnity. Emulation of chemical stimulus triggered head movement in the c. elegans nematode. Neurocomputing, 290:60-73, 2018.

[19] Dávid Csercsik, Imre Farkas, Gábor Szederkényi, Erik Hrabovszky, Zsolt Liposits, and Katalin M Hangos. Hodgkin-huxley type modelling and parameter estimation of gnrh neurons. Biosystems, 100(3):198-207, 2010.

[20] Dávid Csercsik, Katalin M Hangos, and Gábor Szederkényi. Identifiability analysis and parameter estimation of a single hodgkin-huxley type voltage dependent ion channel under voltage step measurement conditions. Neurocomputing, 77(1):178-188, 2012. 
[21] Swagatam Das and Ponnuthurai Nagaratnam Suganthan. Differential evolution: A survey of the state-of-the-art. IEEE transactions on evolutionary computation, 15(1):4-31, 2010.

[22] Swagatam Das, Sankha Subhra Mullick, and Ponnuthurai N Suganthan. Recent advances in differential evolution-an updated survey. Swarm and evolutionary computation, 27:1-30, 2016 .

[23] Peter Dayan and Laurence F Abbott. Theoretical neuroscience: computational and mathematical modeling of neural systems. Computational Neuroscience Series, 2001.

[24] Mario de Bono and Andres Villu Maricq. Neuronal substrates of complex behaviors in c. elegans. Annu. Rev. Neurosci., 28:451-501, 2005.

[25] Shaul Druckmann, Yoav Banitt, Albert A Gidon, Felix Schürmann, Henry Markram, and Idan Segev. A novel multiple objective optimization framework for constraining conductance-based neuron models by experimental data. Frontiers in neuroscience, 1:1, 2007.

[26] Agoston E Eiben, James E Smith, et al. Introduction to evolutionary computing, volume 53. Springer, 2003.

[27] Steffen E Eikenberry and Vasilis Z Marmarelis. Principal dynamic mode analysis of the hodgkin-huxley equations. International journal of neural systems, 25(02):1550001, 2015.

[28] Lesley Emtage, Sonya Aziz-Zaman, Olivia Padovan-Merhar, H Robert Horvitz, Christopher Fang-Yen, and Niels Ringstad. Irk-1 potassium channels mediate peptidergic inhibition of caenorhabditis elegans serotonin neurons via a go signaling pathway. Journal of Neuroscience, 32(46):16285-16295, 2012.

[29] Vitaliy Feoktistov. Differential evolution. Springer, 2006.

[30] Richard FitzHugh. Mathematical models of excitation and propagation in nerve. Biological engineering, pages 1-85, 1969.

[31] Fabio Galán-Prado, Alejandro Morán, Joan Font, Miquel Roca, and Josep L Rosselló. Compact hardware synthesis of stochastic spiking neural networks. International journal of neural systems, 29(08):1950004, 2019.

[32] Alice Geminiani, Claudia Casellato, Alberto Antonietti, Egidio D'Angelo, and Alessandra Pedrocchi. A multiple-plasticity spiking neural network embedded in a closed-loop control system to model cerebellar pathologies. International journal of neural systems, 28(05): $1750017,2018$.

[33] William C Gerken, LK Purvis, and Robert J Butera. Genetic algorithm for optimization and specification of a neuron model. Neurocomputing, 69(10-12):1039-1042, 2006.

[34] Sayan Ghosh, Swagatam Das, Athanasios V Vasilakos, and Kaushik Suresh. On convergence of differential evolution over a class of continuous functions with unique global optimum. IEEE Transactions on Systems, Man, and Cybernetics, Part B (Cybernetics), 42(1):107$124,2011$.

[35] Samanwoy Ghosh-Dastidar and Hojjat Adeli. Improved spiking neural networks for eeg classification and epilepsy and seizure detection. Integrated Computer-Aided Engineering, 14(3):187-212, 2007.

[36] Samanwoy Ghosh-Dastidar and Hojjat Adeli. Spiking neural networks. International journal of neural systems, 19(04):295-308, 2009. 
[37] Samanwoy Ghosh-Dastidar and Hojjat Adeli. A new supervised learning algorithm for multiple spiking neural networks with application in epilepsy and seizure detection. Neural networks, 22(10):1419-1431, 2009.

[38] Jose Gomez-Tames, Akimasa Hirata, Manabu Tamura, and Yoshihiro Muragaki. Corticomotoneuronal model for intraoperative neurophysiological monitoring during direct brain stimulation. International journal of neural systems, 29(01):1850026, 2019.

[39] Miriam B Goodman, David H Hall, Leon Avery, and Shawn R Lockery. Active currents regulate sensitivity and dynamic range in c. elegans neurons. Neuron, 20(4):763-772, 1998.

[40] Andrew Gordus, Navin Pokala, Sagi Levy, Steven W Flavell, and Cornelia I Bargmann. Feedback from network states generates variability in a probabilistic olfactory circuit. Cell, 161(2):215-227, 2015.

[41] D Haufler, F Morin, JC Lacaille, and Frances K Skinner. Parameter estimation in singlecompartment neuron models using a synchronization-based method. Neurocomputing, 70 (10-12):1605-1610, 2007.

[42] Randall D Hayes, John H Byrne, Steven J Cox, and Douglas A Baxter. Estimation of single-neuron model parameters from spike train data. Neurocomputing, 65:517-529, 2005.

[43] Michael Hendricks, Heonick Ha, Nicolas Maffey, and Yun Zhang. Compartmentalized calcium dynamics in a c. elegans interneuron encode head movement. Nature, 487(7405): 99-103, 2012.

[44] Hiroshi Hibino, Atsushi Inanobe, Kazuharu Furutani, Shingo Murakami, IAN Findlay, and Yoshihisa Kurachi. Inwardly rectifying potassium channels: their structure, function, and physiological roles. Physiological reviews, 90(1):291-366, 2010.

[45] James L Hindmarsh and RM Rose. A model of neuronal bursting using three coupled first order differential equations. Proceedings of the Royal society of London. Series B. Biological sciences, 221(1222):87-102, 1984.

[46] Alan L Hodgkin and Andrew F Huxley. A quantitative description of membrane current and its application to conduction and excitation in nerve. The Journal of physiology, 117 (4):500-544, 1952.

[47] Alan L Hodgkin, Andrew F Huxley, and Bernard Katz. Measurement of current-voltage relations in the membrane of the giant axon of loligo. The Journal of physiology, 116(4): 424-448, 1952.

[48] Allan L Hodgkin and Andrew F Huxley. Currents carried by sodium and potassium ions through the membrane of the giant axon of loligo. The Journal of physiology, 116(4): 449-472, 1952.

[49] Allan L Hodgkin and Andrew F Huxley. The components of membrane conductance in the giant axon of loligo. The Journal of physiology, 116(4):473-496, 1952.

[50] Allan L Hodgkin and Andrew F Huxley. The dual effect of membrane potential on sodium conductance in the giant axon of loligo. The Journal of physiology, 116(4):497-506, 1952.

[51] Sture Holm. A simple sequentially rejective multiple test procedure. Scandinavian journal of statistics, pages 65-70, 1979.

[52] Ruihan Hu, Qijun Huang, Hao Wang, Jin He, and Sheng Chang. Monitor-based spiking recurrent network for the representation of complex dynamic patterns. International journal of neural systems, 29(08):1950006, 2019. 
[53] Elisabetta Iavarone, Jane Yi, Ying Shi, Bas-Jan Zandt, Christian O'reilly, Werner Van Geit, Christian Rössert, Henry Markram, and Sean L Hill. Experimentally-constrained biophysical models of tonic and burst firing modes in thalamocortical neurons. PLOS Computational Biology, 15(5):e1006753, 2019.

[54] Eugene M Izhikevich. Dynamical systems in neuroscience. MIT press, 2007.

[55] Eduardo J Izquierdo. Role of simulation models in understanding the generation of behavior in c. elegans. Current Opinion in Systems Biology, 13:93-101, 2019.

[56] James Kunert, Eli Shlizerman, and J Nathan Kutz. Low-dimensional functionality of complex network dynamics: Neurosensory integration in the caenorhabditis elegans connectome. Physical Review E, 89(5):052805, 2014.

[57] James M Kunert, Joshua L Proctor, Steven L Brunton, and J Nathan Kutz. Spatiotemporal feedback and network structure drive and encode caenorhabditis elegans locomotion. PLoS computational biology, 13(1), 2017.

[58] Masahiro Kuramochi and Motomichi Doi. A computational model based on multi-regional calcium imaging represents the spatio-temporal dynamics in a caenorhabditis elegans sensory neuron. PLoS One, 12(1):e0168415, 2017.

[59] Jack Lee, Bruce Smaill, and Nicolas Smith. Hodgkin-huxley type ion channel characterization: an improved method of voltage clamp experiment parameter estimation. Journal of theoretical biology, 242(1):123-134, 2006.

[60] Qiang Liu, Philip B Kidd, May Dobosiewicz, and Cornelia I Bargmann. C. elegans awa olfactory neurons fire calcium-mediated all-or-none action potentials. Cell, 175(1):57-70, 2018.

[61] Maria Markaki and Nektarios Tavernarakis. Modeling human diseases in caenorhabditis elegans. Biotechnology journal, 5(12):1261-1276, 2010.

[62] C Daniel Meliza, Mark Kostuk, Hao Huang, Alain Nogaret, Daniel Margoliash, and Henry DI Abarbanel. Estimating parameters and predicting membrane voltages with conductance-based neuron models. Biological cybernetics, 108(4):495-516, 2014.

[63] Ferrante Neri and Ville Tirronen. Recent advances in differential evolution: a survey and experimental analysis. Artificial intelligence review, 33(1):61-106, 2010.

[64] Martina Nicoletti, Alessandro Loppini, Letizia Chiodo, Viola Folli, Giancarlo Ruocco, and Simonetta Filippi. Biophysical modeling of c. elegans neurons: Single ion currents and whole-cell dynamics of awcon and rmd. PloS one, 14(7), 2019.

[65] Beverly J Piggott, Jie Liu, Zhaoyang Feng, Seth A Wescott, and XZ Shawn Xu. The neural circuits and synaptic mechanisms underlying motor initiation in c. elegans. Cell, 147(4): 922-933, 2011.

[66] H Press. Genome sequence of the nematode c. elegans: a platform for investigating biology. Science, 282:2012-2018, 1998.

[67] Kenneth Price, Rainer M Storn, and Jouni A Lampinen. Differential evolution: a practical approach to global optimization. Springer Science \& Business Media, 2006.

[68] Franciszek Rakowski, Jagan Srinivasan, Paul W Sternberg, and Jan Karbowski. Synaptic polarity of the interneuron circuit controlling c. elegans locomotion. Frontiers in computational neuroscience, 7:128, 2013. 
[69] Kerrianne Ryan, Zhiyuan Lu, and Ian A Meinertzhagen. The cns connectome of a tadpole larva of ciona intestinalis (1.) highlights sidedness in the brain of a chordate sibling. Elife, 5:e16962, 2016.

[70] Kazumi Sakata and Ryuzo Shingai. Neural network model to generate head swing in locomotion of caenorhabditis elegans. Network: Computation in Neural Systems, 15(3): 199-216, 2004.

[71] Bert Sakmann. Single-channel recording. Springer Science \& Business Media, 2013.

[72] Lawrence B Salkoff, Aguan Wei, Beravan Baban, Alice Butler, Gloria L Fawcett, Gonzalo Ferreira, and Celia M Santi. Potassium channels in c. elegans. WormBook, 2005.

[73] Rahul Sarpeshkar. Analog versus digital: extrapolating from electronics to neurobiology. Neural computation, 10(7):1601-1638, 1998.

[74] Tomomi Shindou, Mayumi Ochi-Shindou, Takashi Murayama, Ei-ichiro Saita, Yuto Momohara, Jeffery R Wickens, and Ichiro N Maruyama. Active propagation of dendritic electrical signals in c. elegans. Scientific reports, 9(1):1-12, 2019.

[75] Boris Borisovich Shtonda and Leon Avery. Dietary choice behavior in caenorhabditis elegans. Journal of experimental biology, 209(1):89-102, 2006.

[76] Rainer Storn and Kenneth Price. Differential evolution-a simple and efficient heuristic for global optimization over continuous spaces. Journal of global optimization, 11(4):341-359, 1997.

[77] Fei Su, Jiang Wang, Bin Deng, Xi-Le Wei, Ying-Yuan Chen, Chen Liu, and Hui-Yan Li. Adaptive control of parkinson's state based on a nonlinear computational model with unknown parameters. International journal of neural systems, 25(01):1450030, 2015.

[78] Yuki Todo, Zheng Tang, Hiroyoshi Todo, Junkai Ji, and Kazuya Yamashita. Neurons with multiplicative interactions of nonlinear synapses. International journal of neural systems, 29(08):1950012, 2019.

[79] Danièle Tritsch, Dominique Chesnoy-Marchais, and Anne Feltz. Physiologie du neurone. Wolters Kluwer France, 1999.

[80] Ivan Tyukin, Erik Steur, Henk Nijmeijer, David Fairhurst, Inseon Song, Alexey Semyanov, and CEES VAN LEEUWEN. State and parameter estimation for canonic models of neural oscillators. International journal of neural systems, 20(03):193-207, 2010.

[81] Lav R Varshney, Beth L Chen, Eric Paniagua, David H Hall, and Dmitri B Chklovskii. Structural properties of the caenorhabditis elegans neuronal network. PLoS computational biology, 7(2), 2011.

[82] Siva Venkadesh, Alexander O Komendantov, Stanislav Listopad, Eric O Scott, Kenneth De Jong, Jeffrey L Krichmar, and Giorgio A Ascoli. Evolving simple models of diverse intrinsic dynamics in hippocampal neuron types. Frontiers in neuroinformatics, 12:8, 2018.

[83] Jakob Vesterstrom and Rene Thomsen. A comparative study of differential evolution, particle swarm optimization, and evolutionary algorithms on numerical benchmark problems. In Proceedings of the 2004 congress on evolutionary computation (IEEE Cat. No. 04TH8753), volume 2, pages 1980-1987. IEEE, 2004.

[84] John G White, Eileen Southgate, J Nichol Thomson, and Sydney Brenner. The structure of the nervous system of the nematode caenorhabditis elegans. Philos Trans $R$ Soc Lond B Biol Sci, 314(1165):1-340, 1986. 
[85] Stephen R Wicks, Chris J Roehrig, and Catharine H Rankin. A dynamic network simulation of the nematode tap withdrawal circuit: predictions concerning synaptic function using behavioral criteria. Journal of Neuroscience, 16(12):4017-4031, 1996.

[86] Frank Wilcoxon. Individual comparisons by ranking methods. In Breakthroughs in statistics, pages 196-202. Springer, 1992.

[87] Allan R Willms, Deborah J Baro, Ronald M Harris-Warrick, and John Guckenheimer. An improved parameter estimation method for hodgkin-huxley models. Journal of computational neuroscience, 6(2):145-168, 1999.

[88] Knut M Wittkowski. Friedman-type statistics and consistent multiple comparisons for unbalanced designs with missing data. Journal of the American Statistical Association, 83 (404):1163-1170, 1988.

[89] Andrew P Wojtovich, Peter DiStefano, Teresa Sherman, Paul S Brookes, and Keith Nehrke. Mitochondrial atp-sensitive potassium channel activity and hypoxic preconditioning are independent of an inwardly rectifying potassium channel subunit in caenorhabditis elegans. FEBS letters, 586(4):428-434, 2012.

[90] Tingfang Wu, Florin-Daniel Bîlbîe, Andrei Păun, Linqiang Pan, and Ferrante Neri. Simplified and yet turing universal spiking neural $\mathrm{p}$ systems with communication on request. International journal of neural systems, 28(08):1850013, 2018.

[91] Guosheng Yi, Jiang Wang, Xile Wei, Bin Deng, Kai-Ming Tsang, Wai-Lok Chan, and Chunxiao Han. Effects of extremely low-frequency magnetic fields on the response of a conductance-based neuron model. International journal of neural systems, 24(01):1450007, 2014 .

[92] Dingguo Zhang, Qing Zhang, and Xiangyang Zhu. Exploring a type of central pattern generator based on hindmarsh-rose model: from theory to application. International journal of neural systems, 25(01):1450028, 2015.

[93] Xu Zhang, Greg Foderaro, Craig Henriquez, and Silvia Ferrari. A scalable weight-free learning algorithm for regulatory control of cell activity in spiking neuronal networks. International journal of neural systems, 28(02):1750015, 2018.

[94] Maohua Zheng, Pengxiu Cao, Jiong Yang, XZ Shawn Xu, and Zhaoyang Feng. Calcium imaging of multiple neurons in freely behaving c. elegans. Journal of neuroscience methods, 206(1):78-82, 2012.

[95] Zhihao Zheng, J Scott Lauritzen, Eric Perlman, Camenzind G Robinson, Matthew Nichols, Daniel Milkie, Omar Torrens, John Price, Corey B Fisher, Nadiya Sharifi, et al. A complete electron microscopy volume of the brain of adult drosophila melanogaster. Cell, 174(3):730$743,2018$. 